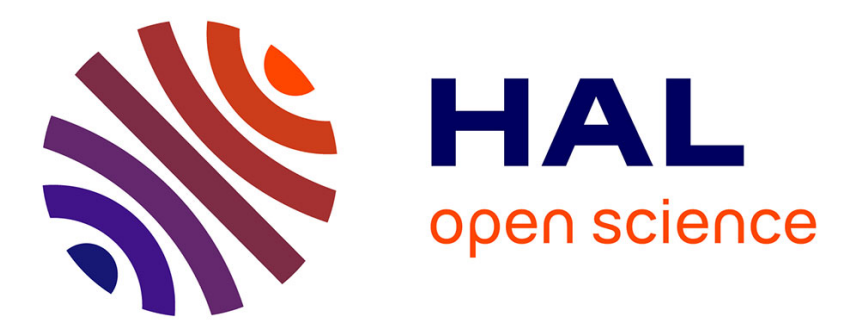

\title{
Multiple-band OFDM transmission exploiting the frequency chirping properties of Silicon Ring-Resonator Modulators
}

Mohamed E Chaibi, Olivier Dubray, Laurent Bramerie, Karim Hassan, Christophe Peucheret

\section{To cite this version:}

Mohamed E Chaibi, Olivier Dubray, Laurent Bramerie, Karim Hassan, Christophe Peucheret. Multiple-band OFDM transmission exploiting the frequency chirping properties of Silicon RingResonator Modulators. ACP 2017 - Asia Communications and Photonics Conference, Nov 2017, Guangzhou, China. hal-01633242

\section{HAL Id: hal-01633242 https://hal.science/hal-01633242}

Submitted on 12 Nov 2017

HAL is a multi-disciplinary open access archive for the deposit and dissemination of scientific research documents, whether they are published or not. The documents may come from teaching and research institutions in France or abroad, or from public or private research centers.
L'archive ouverte pluridisciplinaire HAL, est destinée au dépôt et à la diffusion de documents scientifiques de niveau recherche, publiés ou non, émanant des établissements d'enseignement et de recherche français ou étrangers, des laboratoires publics ou privés. 


\title{
Multiple-Band OFDM Transmission Exploiting the Frequency Chirping Properties of Silicon Ring-Resonator Modulators
}

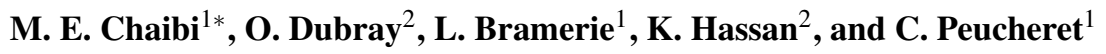 \\ 1-FOTON Laboratory, CNRS, University of Rennes 1, 22300 Lannion, France \\ 2-University Grenoble Alpes, CEA, LETI, MINATEC Campus, 38054 Grenoble, France \\ *chaibi@enssat.fr
}

Abstract: The complementarity of the frequency responses of IM-DD channels for positive and negative detunings of ring-resonator modulators, obtained as a result of their chirping properties, is exploited to overcome frequency fading limitations in DD-OFDM systems.

\section{Introduction}

OCIS codes: (060.4510) Optical communications; (060.4080) Modulation; (130.3120) Integrated optics devices.

Constraints in terms of cost, power consumption and device footprint have to be considered when upgrading the capacities of optical access networks to $40 \mathrm{~Gb} / \mathrm{s}$ and beyond. In addition, future optical access networks must extend their reaches to as long as $100 \mathrm{~km}$ to accommodate more end-users and decrease the number of required central offices. Already-installed optical access systems are typically based on intensity modulation (IM) and direct detection (DD), with traditional non-return-to-zero (NRZ) modulation. In order to achieve higher bit rates and still utilize low-cost and low-bandwidth modulators, highly spectrally-efficient modulation formats have to be adopted. Orthogonal frequency division multiplexing (OFDM) modulation is particularly interesting in access networks thanks to its scalability and dynamic multiple access capability [1]. However, the use of high-bit rate conventional double-sideband OFDM over long-distances is limited due to the occurrence of channel fading that results from dispersion-induced frequencydependent phase-shifts experienced by the subcarriers and direct-detection. The presence of transient frequency chirp originating from the modulation process further limits the capacity $\times$ distance product in such systems.

Silicon ring-resonator modulators (RRMs) comply with future access networks cost, energy-efficiency and footprint requirements. Wide-bandwidth and high-extinction ratio modulators exploiting carrier-depletion in PN junctions have been demonstrated. The frequency-chirping properties of RRMs have, however, only been the subject of a limited number of studies to date $[2,3]$ and have not been fully investigated yet. Very few demonstrations of subcarrier multiplexing [4], including discrete multi-tone (DMT) modulation [5] and OFDM [6] employing silicon RRMs have been reported so far. Channel frequency fading due to fiber dispersion has limited transmission to a few $10^{\prime}$ s of kilometers. In this paper, we demonstrate and propose to exploit the different frequency-chirping properties of a silicon RRM on each side of its resonance, which result in complementary channel frequency responses, in order to increase the capacity $\times$ distance product of DD-OFDM systems. We first thoroughly characterize the frequency chirp in RRMs and its impact on the channel frequency response before proposing a novel multiple-band transmitter architecture that overcomes the channel fading effect. In a proof-of-concept experiment, we demonstrate multiple-band OFDM transmission at $15.15 \mathrm{~Gb} / \mathrm{s}$ over $100 \mathrm{~km}$ standard single-mode fiber (SSMF).

\section{Frequency-chirping in RRMs}

A small-signal modulation model describing RRMs operating in carrier depletion [7] can be extended to model their frequency chirping behaviour. In particular, an analytical expression can be derived for the ratio $2 \beta / m$ of the phase modulation (PM) index $\beta$ to the intensity modulation (IM) index $m$ under small-signal modulation as a function of the modulation frequency. The phase-shift between IM and PM can be accounted for by considering $2 \beta / \mathrm{m}$ as a complex parameter. Based on parameters extracted from static and small-signal characterizations of RRMs [7], the modulus and phase of $2 \beta / \mathrm{m}$ are calculated and represented as a function of modulation frequency in Fig. 1(a)-(b) for different values of the wavelength detuning between the continuous-wave $(\mathrm{CW})$ laser wavelength and the RRM resonance wavelength, $\Delta \lambda=\lambda_{0}-\lambda_{C W}$, which will be referred to as "wavelength detuning" throughout the paper. It can be seen in Fig. 1(a) that the chirp of RRMs is strongly dependent on the modulation frequency. In addition, the wavelength detuning clearly affects the chirp coefficient. Moving from positive to negative detuning induces a $\sim \pi$ jump in the relative phase-shift between IM and PM, as seen in Fig. 1(b). This has an important effect on the frequency response of an IM-DD channel, as illustrated in Fig. 1(c) for transmission over $100 \mathrm{~km}$ SSMF. The fading frequencies can be shifted by merely changing the wavelength detuning value from positive to negative. An interesting configuration is when two complementary frequency responses exist, where the fading frequencies obtained for a positive value of $\Delta \lambda$ 


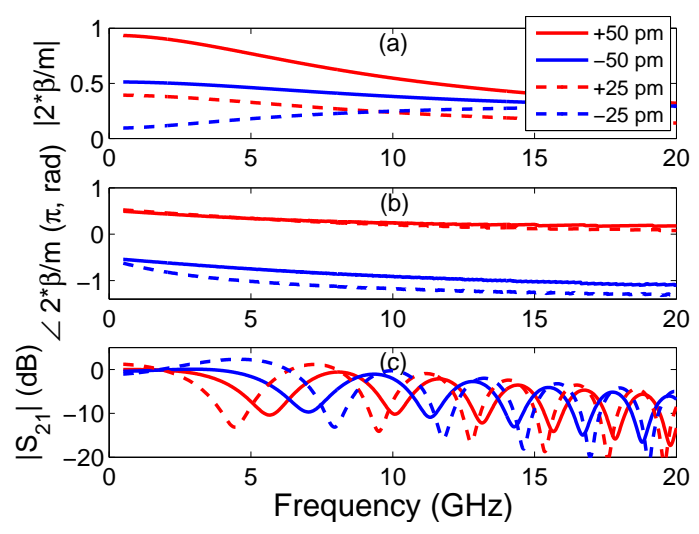

Fig. 1: Calculated modulus and phase of $2 \beta / \mathrm{m}$ and frequency responses of an $100 \mathrm{~km}$ SSMF link as a function of modulation frequency for different $\Delta \lambda$.

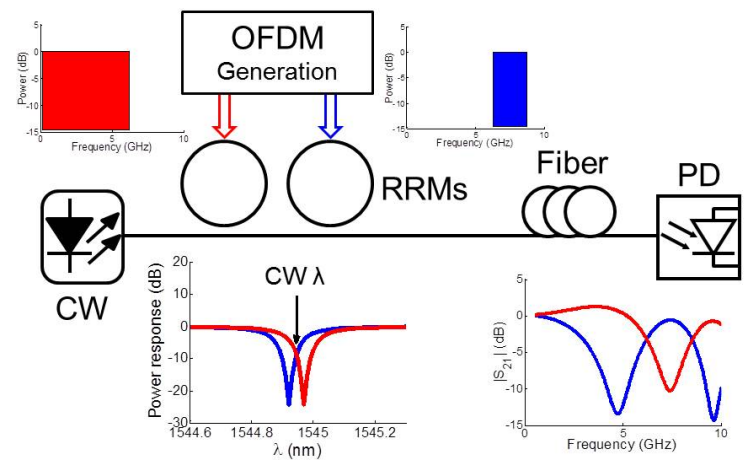

Fig. 3: Principle of the proposed technique.
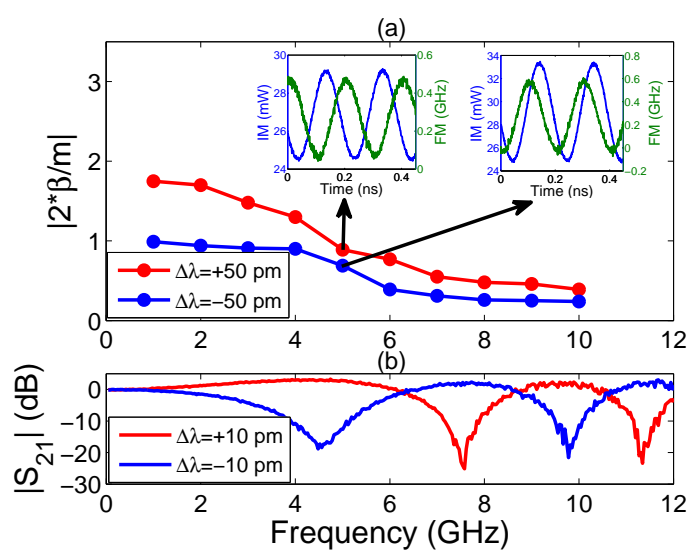

Fig. 2: (a) Measured $2 \beta / m$ as a function of modulation frequency for different RRM detunings and (b) example of complementary frequency responses over $100 \mathrm{~km}$.

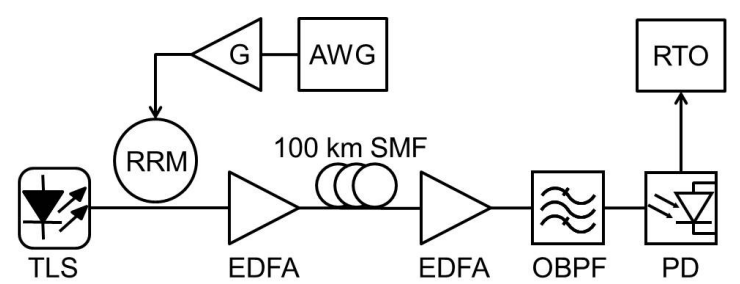

Fig. 4: Experimental setup. AWG: arbitrary waveform generator; EDFA: erbium-doped fiber amplifier; OBPF: optical bandpass filter.

coincide with the maxima of the response obtained for negative values of $\Delta \lambda$. These complementary responses will be exploited to overcome the frequency fading limitations in OFDM transmission. Based on dynamic chirp characterizations, $2 \beta / m$ was evaluated on a silicon RRM for different $\Delta \lambda$ values. The measurements reported in Fig. 2(a) confirm the theoretical predictions regarding the modulation frequency dependence of the chirp. Furthermore, the insets of Fig. 2(a), representing the measured time dependence of the intensity and frequency for $5 \mathrm{GHz}$ modulation and $\Delta \lambda= \pm 50 \mathrm{pm}$ confirm the existence of a $\pi$ shift between the relative PM and IM responses on either side of the resonance. The experimental channel responses measured over 100-km SSMF shown in Fig. 2(b) confirm the possibility of obtaining complementary channel responses for negative and positive wavelength detunings.

The complementarity between channel responses associated to positive and negative detunings of the RRM resonance with respect to the laser frequency is exploited in a novel OFDM transmitter architecture, whose principle is schematically represented in Fig. 3. The electrical OFDM signal is divided into sub-bands that are applied to one of two distinct RRMs in series biased so that their detuning with respect to the CW laser wavelength is either positive or negative. The OFDM sub-bands allocation to each RRM is made so that they do not overlap with the fading regions after propagation over a dispersive channel.

\section{Experimental setup}

The experimental set-up used for the proof-of-concept is represented in Fig. 4. In the absence of a dual-RRM chip, the same RRM is used sequentially with positive and negative detunings. The modulating signal consists of 300 OFDM symbols distributed over 250 out of a total of 256 subcarriers modulated with quadrature phase-shift keying (QPSK) data, and 8 samples being used as cyclic prefix. The analogue OFDM signal, which occupies a 8 GHz bandwidth and whose overall bit rate is $15.15 \mathrm{~Gb} / \mathrm{s}$, is amplified and used to modulate the RRM. A tunable laser source (TLS) allows an easy adjustment of the wavelength detuning to $\Delta \lambda= \pm 10 \mathrm{pm}$, which constitutes a trade-off between the RRM bandwidth and the optical modulation amplitude (OMA). The optical modulated signal is then boosted and transmitted 
(a)

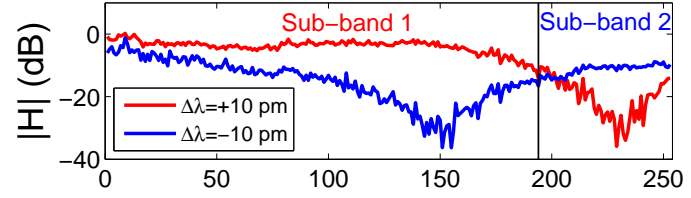

(b)

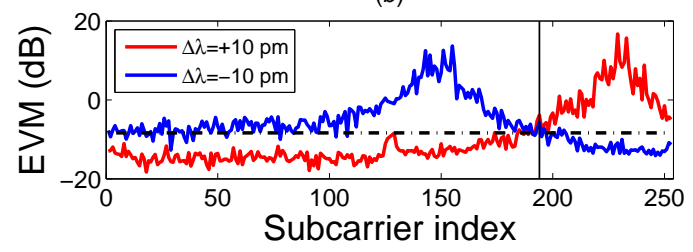

Fig. 5: (a) Channel responses and (b) EVM per OFDM subcarrier after $100 \mathrm{~km} \mathrm{SSMF}$ for $\Delta \lambda= \pm 10$ pm.

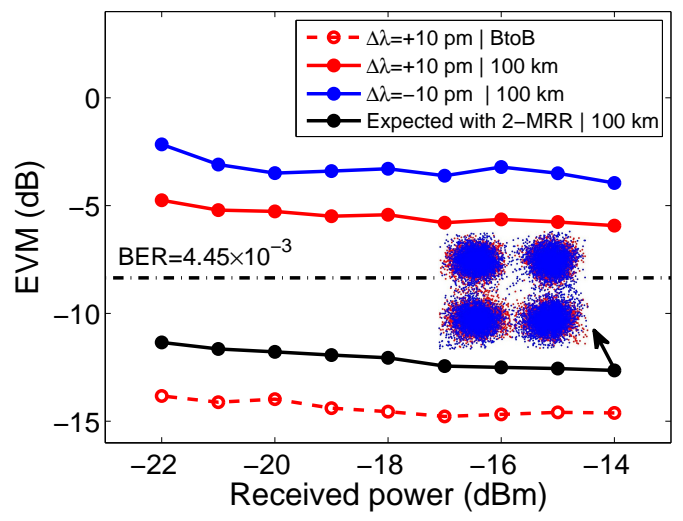

Fig. 6: EVM averaged over all subcarriers for B2B and after $100 \mathrm{~km}$ SSMF, as well as averaged taking into account their distribution into two sub-bands applied to 2 RRMs.

over $100 \mathrm{~km}$ SSMF before being pre-amplified, bandpass-filtered to eliminate amplified spontaneous emission noise and photo-detected. A real-time oscilloscope (RTO) implements analog-to-digital conversion at $40 \mathrm{GS} / \mathrm{s}$ sampling frequency. The OFDM signals are then processed offline using Matlab. To show the impact of the complementary channel responses, the entire OFDM signal is applied to the RRM, while in practice it would be divided in multiple bands applied to the two RRMs.

\section{Results and discussions}

The channel responses estimated from the OFDM subcarriers and the error vector magnitude (EVM) per OFDM sub-carrier are shown in Fig. 5 for $-15 \mathrm{dBm}$ received power at the input of the pre-amplifier and for $\Delta \lambda= \pm 10$ pm, confirming their complementary nature. A large dispersion of the EVM values is clearly visible in Fig. 5 (b) for the two wavelength detunings. We remind here that the whole OFDM signal is sequentially applied to the RRM for the two detuning values, while in our proposed architecture, it will be divided into sub-bands. By dividing the OFDM signal into 2 bands, as shown in Fig. 5(a), up to 97\% of the subcarriers keep their EVM value below -8.35 dB, corresponding to a BER threshold of $4.45 \times 10^{-3}$ (7\% forward error correction overhead) after $100 \mathrm{~km} \mathrm{SSMF.} \mathrm{In} \mathrm{Fig.} \mathrm{6,} \mathrm{the} \mathrm{mean}$ values of EVM are plotted as a function of received power after $100 \mathrm{~km}$ SSMF for $\pm 10 \mathrm{pm}$ detuning. The backto-back (B2B) performance at $+10 \mathrm{pm}$ is used as a reference. First, EVM values averaged over all subcarriers are represented after $100 \mathrm{~km}$ for detunings of $+10 \mathrm{pm}$, then $-10 \mathrm{pm}$. High penalties compared to the B2B reference case are obtained and the EVM threshold of $-8.35 \mathrm{~dB}$ is not reached. Next, the EVM is calculated based on a distribution of the subcarriers into 2 frequency bands, which are applied to the RRM with negative and positive detunings according to the allocation shown in Fig. 5(a). This therefore represents the EVM that is expected from the use of 2 separate RRMs. In this case, EVM values close to the B2B case are measured, confirming the good performance of our proposed scheme.

\section{Conclusions}

The chirp properties of silicon MRR modulators have been studied and exploited in a novel OFDM transmitter architecture to overcome frequency fading limitations when propagating over a dispersive channel. It has been shown that a two-MRR structure could allow OFDM transmission at $15.15 \mathrm{~Gb} / \mathrm{s}$ over $100 \mathrm{~km} \mathrm{SSMF}$.

\section{References}

1. X. Q. Jin and J. M. Tang, Proc. OFC, OWA4B.5, Los Angeles (2012).

2. O. Dubray and S. Menezo, IEEE Photon. Technol. Lett. 28, 280-283 (2016).

3. Z. Wang et al., J. Lightw. Technol. 34, 3675-3681 (2016).

4. Y. Gao et al., Proc. OFC, Th1B.2, Los Angeles (2017).

5. P. Dong et al., Proc. OFC, W4J.4, Anaheim (2016).

6. X. Wu et al., IEEE Photon. Technol. Lett. 28, 2058-2061 (2016).

7. H. Yu et al., Opt. Express 22, 15178-15189 (2014). 